\title{
Acceptability and Usability of HCV Self-Testing in High Risk Populations in Vietnam
}

\author{
Linh Thuy Nguyen ${ }^{1, \dagger}$, Van Thi Thuy Nguyen ${ }^{2, *},+\mathbb{D}$, Kim Anh Le Ai ${ }^{3}$, Minh Binh Truong ${ }^{3}$, Tam Thi Minh Tran ${ }^{1}$, \\ Muhammad S. Jamil ${ }^{4}{ }^{\infty}$, Cheryl Johnson ${ }^{4}$, Elena Ivanova Reipold ${ }^{5}$, Philippa Easterbrook ${ }^{4}$ and Kidong Park ${ }^{2}\left({ }^{1}\right.$ \\ 1 Center for Bio-Medical Research, National Institute of Hygiene and Epidemiology, Hanoi 100000, Vietnam; \\ linh.nguyen.2@ucdconnect.ie (L.T.N.); tamtranskku@gmail.com (T.T.M.T.) \\ 2 Country Office in Viet Nam, World Health Organization, Hanoi 100000, Vietnam; parkk@who.int \\ 3 Thai Nguyen Provincial Centre for Disease Control, Thai Nguyen 24000, Vietnam; \\ laka0280@gmail.com (K.A.L.A.); bsminhcdtdltn@gmail.com (M.B.T.) \\ 4 Global HIV, Hepatitis and STI Programmes, World Health Organization, 1202 Geneva, Switzerland; \\ mjamil@who.int (M.S.J.); johnsonc@who.int (C.J.); easterbrookp@who.int (P.E.) \\ 5 Foundation for Innovative New Diagnostics, 1202 Geneva, Switzerland; Elena.Ivanova@finddx.org \\ * Correspondence: nguyenva@who.int; Tel.: +84-2438500314 \\ + These authors contributed equally to this work.
}

\section{check for} updates

Citation: Nguyen, L.T.; Nguyen, V.T.T.; Le Ai, K.A.; Truong, M.B.; Tran, T.T.M.; Jamil, M.S.; Johnson, C.; Reipold, E.I.; Easterbrook, P.; Park, K. Acceptability and Usability of HCV Self-Testing in High Risk Populations in Vietnam. Diagnostics 2021, 11, 377. https://doi.org/10.3390/ diagnostics11020377

Academic Editors: Elisa Martró and Tanya Applegate

Received: 5 December 2020

Accepted: 19 February 2021

Published: 23 February 2021

Publisher's Note: MDPI stays neutral with regard to jurisdictional claims in published maps and institutional affiliations.

Copyright: (c) 2021 by the authors. Licensee MDPI, Basel, Switzerland. This article is an open access article distributed under the terms and conditions of the Creative Commons Attribution (CC BY) license (https:/ / creativecommons.org/licenses/by/ $4.0 /)$.

\begin{abstract}
HIV self-testing has emerged as a safe and effective approach to increase the access to and uptake of HIV testing and treatment, especially for key populations. Applying self-testing to hepatitis $\mathrm{C}$ virus (HCV) may also offer an additional way to address low coverage of $\mathrm{HCV}$ testing and to accelerate elimination efforts. To understand the potential for HCV self-testing (HCVST), an observational study was conducted to assess the acceptability and usability of the OraQuick ${ }^{\circledR}$ HCV Self-Test (prototype) among people who inject drugs (PWID) and men who have sex with men (MSM) in Thai Nguyen, a province in northern Vietnam. A total of 105 PWID and 104 MSM were eligible and agreed to participate in the study. Acceptability, defined as the proportion of participants among eligible subjects who agreed to participate in the study, was $92.9 \%$ in PWID and $98.6 \%$ in MSM. Compared to MSM, PWID were older (median age: 45 vs. 22 years; $p<0.0001$ ) and had a lower education level (high school and college: $38.1 \%$ vs. $100 \% ; p<0.0001$ ). HCVST usability was high among MSM with fewer observed mistakes, difficulties, or participants requiring assistance (33.7\%, 28.8\%, and $17.3 \%$, respectively) compared to PWID $(62.9 \%, 53.3 \%$, and $66.7 \%$, respectively; all $p<0.0001)$ ). Inter-reader and inter-operator agreement were good in both groups (Kappa coefficient range: 0.61-0.99). However, the concordance between HCVST and study staff -read or performed HCV testing was lower among PWID than MSM (inter-reader concordance $88.6 \%$ vs. $99.0 \%$ and inter-operator concordance $81.9 \%$ vs. $99 \%$ ). Overall, HCVST was highly acceptable with moderate to high usability among PWID and MSM in Thai Nguyen. Efforts to provide support and assistance may be needed to optimize performance, particularly for PWID populations and for those who are older and with lower literacy or education levels.
\end{abstract}

Keywords: HCV; self-testing; oral fluid; PWID; MSM; Vietnam

\section{Introduction}

Viral hepatitis B and C are major causes of morbidity and mortality worldwide. Together, they lead to approximately 1.34 million deaths per year [1]. In 2015, an estimated 71 million people had chronic hepatitis $\mathrm{C}$ virus $(\mathrm{HCV})$ infection worldwide, and around three-quarters of them lived in low- and middle-income countries [1]. Despite the availability of treatment and a cure for $\mathrm{HCV}$, less than $20 \%$ of people with chronic hepatitis C have been tested and are aware of their infection [1].

Most transmission of HCV in high-income settings, including North America and Western and Eastern Europe, is due to previous or current injecting drug use. In all regions, 
injecting drug users are at higher risk of acquiring HCV than the general population, and two-thirds of people who inject drugs (PWID) are estimated to have been infected with $\mathrm{HCV}$ [2]. A high prevalence of HCV infection among men who have sex with men (MSM) was recently reported in Taiwan with the HCV incidence per 1000 person years increased from 12.9 in 2014 to 25.4 in 2018 [3]. In this study, syphilis infection was associated with HCV seroconversion, which suggested that behaviors such as unprotected anal sex and chemsex can increase the risk of sexually transmitted infections including HCV. Unsafe injection and medical practices also contribute significantly to new infections in many lowand middle-income countries with generalized epidemics. The most affected regions are Eastern Mediterranean region and north and west Africa, where most infections are caused by unsafe medical injections and other medical procedures.

Vietnam is among the 20 countries with the highest burden of chronic HCV infection in the world and PWID are the most affected population. HCV antibody prevalence among the general population in Vietnam ranged from $0.2-3.3 \%$ [4], but it is substantially higher among PWID (74-87\%) [5], HIV-infected individuals (22.9-89.0\%) [6], HIV-infected MSM (83.1-100\%) and HIV-uninfected MSM (8.9-28.2\%) [7], and multi-transfused and dialysis patients (6-26.6\%) [8,9]. A modelling exercise conducted by the Vietnam Ministry of Health and World Health Organization (WHO) in 2017 estimated that there were nearly one million people with chronic HCV infection in Vietnam. Although no disaggregated data are available on HCV infection among high-prevalence key populations, the most affected groups are PWID, MSM, and people who have had chronic hemodialysis and multiple blood transfusions. The national response to viral hepatitis is based on a national action plan on prevention and control of viral hepatitis (2015-2019) that presents a set of comprehensive measures for prevention, alongside testing and treatment. Since 2019, direct-acting antivirals (DAA) have been included in the health insurance reimbursement list. However, the patient copayment rate is still as high as 50\%, resulting in limited access to $\mathrm{HCV}$ treatment. Advocacy for the reduction of patient copayment to $20 \%$ is ongoing.

The recent introduction of DAAs has transformed treatment options for persons with chronic hepatitis C infection, leading to cure in more than $90 \%$ of treated individuals $[10,11]$. The WHO Global Health Sector Strategy on Viral Hepatitis 2016-2021 has a goal to eliminate viral hepatitis C and B infection as a public health threat by 2030 -defined as a reduction in mortality by $65 \%$ and new infections by $90 \%$ [2]. The attainment of this goal will require $90 \%$ of those infected to be diagnosed, and $80 \%$ of those diagnosed to be treated. Therefore, the substantial scale-up of testing and treatment of chronic HCV infection will be required from the current level, where less than $20 \%$ of those infected are diagnosed.

WHO recommends treatment for all $\mathrm{HCV}$-infected persons with pan-genotypic regimens regardless of stage of the disease. WHO also recommends focused testing in the most affected populations. These include those who have a history of exposure or high-risk behaviors for HCV infection (e.g., PWID, people in prisons and other closed settings, MSM, sex workers, HIV-infected persons, and children of mothers with chronic HCV infection especially if co-infected with HIV), as well the general population in settings with an HCV antibody prevalence $\geq 2-5 \%$ [12]. Although testing can be performed using simple and affordable rapid diagnostic tests (RDT), access to HCV testing services and confirmatory $\mathrm{HCV}$ viral load testing remains a major barrier to scale-up of treatment and elimination of the disease. Many of those affected in high HCV burden countries live in rural or remote settings or are members of high-risk marginalized populations such as PWID or MSM that are hard to reach and stigmatized. Consequently, the uptake of HCV testing and treatment in these high-risk populations remains low, and new strategic approaches to increase HCV testing coverage are needed.

WHO recommends a range of $\mathrm{HCV}$ testing approaches, including a combination of simple and affordable RDTs and confirmatory HCV viral load testing, particularly for those most affected by chronic HCV infection [12]. Access to these services, however, is limited in many settings due to stigma and discrimination, particularly for PWID and MSM, who 
also have high HCV prevalence and incidence. New strategic approaches to increase HCV testing coverage especially in key populations such as PWIDs and MSM are needed.

One such approach is self-testing. In self-testing, an individual performs a simple rapid test and interprets their own result, and it has been shown to significantly increase the uptake of HIV testing services [13,14]. Numerous studies have shown self-testing to be safe, accurate, effective and acceptable to many populations unreached by existing services [13,15-17]. Since 2016, WHO has recommended self-testing for HIV and currently lists four WHO prequalified products for self-testing [18,19]. As of July 2020, 88 countries had national policies supporting self-testing and 41 were fully implementing-including Vietnam where HIVST is widely implemented among key populations [20].

As for HIV, self-testing for HCV has potential to increase access to HCV testing for those unreached by existing services and to contribute to efforts to treat, cure, and eliminate $\mathrm{HCV}$ by 2030. To adapt and introduce self-testing for HCV, it is important to understand the usability and acceptability among potential users. Thus, to inform the development of HCV self-testing (HCVST) policy at global, regional, and national levels, we report on findings from an observational study exploring HCVST acceptability and usability among PWID and MSM in Vietnam.

\section{Objective}

The main study objectives were: (1) to determine the acceptability of and preferences for HCVST among populations at high-risk for HCV infection (PWID and MSM) and (2) to determine the usability (the ability to correctly perform test and interpret results) of an HCV self-test.

\section{Material and Methods}

\subsection{Study Design and Sample Size}

This was an observational study. As there was no published data on acceptance and usability of HCV self-testing, we made a conservative assumption that $50 \%$ of eligible individuals will accept self-testing. To estimate the proportion in this study with a $95 \%$ confidence interval based on Wilson's score method, with $\pm 10 \%$ margin of error, a minimum sample size of 100 participants was required.

It was expected that the two groups would screen approximately 200 eligible subjects during the recruitment period and approximately 100 participants would agree to perform the test. In fact, we were able to reach higher number of eligible participants with higher response rate. A total of 209 participants (105 PWID and 104 MSM) were included in the study compared to the target of 100 .

\subsection{Setting and Participant Recruitment}

The study was conducted in Thai Nguyen province, North Vietnam. Thai Nguyen is one of the provinces with a high burden of HIV, and it also has strong networks of PWID and MSM which have led community-based HIV testing since 2017 [20].

Peer educators recruited participants by reaching out to PWID and MSM communities and using a short questionnaire to identify eligible individuals. The recruitment took place at the offices of the three community-based organizations (two for PWID and one for MSM) where community-based- and key population-led HIV testing is provided. In addition, an online approach was also applied through social media (Facebook, Zalo, etc.).

Those eligible were invited to participate in the study, with separate sessions organized for PWID and MSM, and offered an OraQuick HCV self-test kit (OraSure Technologies Inc., Bethlehem, PA, USA). Those who agreed to participate provided a written informed consent prior to study enrollment. Individuals with reactive results were referred to further testing and treatment at a nearby facility according to the national guidance. 


\subsection{Study Population-Eligibility Criteria}

Individuals who self-identified as PWID and/or MSM were considered eligible and enrolled in the study if they were $\geq 18$ years old, were able to provide written informed consent, had negative or unknown HCV status in the past 12 months, were able to read Vietnamese, and had no previous experience self-testing for HIV and/or HCV. PWID enrolled also had to report use of un-prescribed intravenous drugs within the past 12 months. MSM enrolled also had to report at least one anal sex episode with another man within the past 12 months.

\subsection{Study Procedures}

Baseline information on demographic characteristics, exposure to risk factors, and previous experience with $\mathrm{HCV}$ testing services was collected via questionnaires. The study participants were provided with written and pictorial instructions in Vietnamese on how to perform the HCVST and then were asked to perform the test and interpret the result. The HCVST kit contained: one OraQuick ${ }^{\circledR}$ HCV Rapid Antibody Test (OraSure Technologies Inc., Bethlehem, PA, USA), developer solution, test stand (plastic), desiccant, and manufacturer-provided instructions for use. While the OraQuick ${ }^{\circledR}$ HCV Rapid Antibody Test is prequalified by WHO for professional use, all participants were informed that the kit and instructions for self-testing were for research purposes only.

HCVST was performed at community-based organization offices-where communitybased testing for HIV is regularly conducted. In these key population-led testing sites, 5 principles of HIV testing including privacy were applied. Same principles were applied for HCVST.

A study staff member silently observed the participants and documented any error in testing procedure using a standardized checklist. The study staff did not provide any assistance unless specifically requested by a participant or after multiple efforts to conduct the test unassisted.

After self-testing was completed and the participants read their results, the test result was re-read by a study staff member to measure inter-reader concordance. Then, a brief post-testing interview was conducted to collect acceptability and preferences. Following this, to measure inter-operator concordance, we compared the self-tester's results to a second OraQuick ${ }^{\circledR}$ HCV Rapid Antibody Test that was performed by a study staff member who was blinded to the self-reported results.

Post-test counselling was provided by study staff from provincial Centre for Disease Control. The key messages provided to individuals with reactive results were the meaning of HCVST positive results, the necessity of retesting in health facility, the benefit of HCV treatment, and the location of the health facility where participants could access $\mathrm{HCV}$ testing, diagnosis, and treatment in Thai Nguyen province.

\subsection{Data Analysis}

The results are reported as percentage and median (range) as appropriate. The statistical significance of differences between groups was analyzed by the Fisher's exact test and Chi square test, and $p$ values less than 0.05 were considered statistically significant. Usability was assessed by calculating the frequencies of mistakes, difficulties and assistance needed at each step of the testing procedure from opening the package to reading the results. Inter-reader concordance for self-test results was defined as agreement between the results interpreted by the participant and by a study staff member, reported as a percentage. Inter-operator concordance was defined as agreement between the results of the self-testing reported by the participant and results of professional use test conducted by a study staff member, reported as a percentage. Cohen's Kappa statistic was calculated for both inter-reader and inter-operator concordance. When there was only one rating, Gwet's AC coefficient was used to assess level of agreement. Acceptability and attitudes about self-testing were reported as frequencies and described descriptively. We also used Chi-square test to explore the factors associated with user errors. The statistical analysis 
was performed with software MedCalc version 14.8.1 (MedCalc Software Ltd, Acacialaan, Ostend, Belgium).

\subsection{Ethical Approval}

The Review Board in Bio-medical Research at National Institute of Hygiene and Epidemiology, Hanoi, Vietnam approved the study on 30 July 2019 (IRB-VN01057/IORG 0008555, number NIHE IRB—20/2019).

\section{Results}

\subsection{Patient Recruitment and Characteristics}

A total of 327 individuals (182 PWID and 145 MSM) were approached and screened for eligibility. Among 282 eligible participants (141 PWID and 141 MSM), 270 (95.7\%) agreed to participate in the study (131 PWID and 139 MSM). However, due to the time delay between agreement and enrollment, only $77.4 \%$ (209/270) participants were available and enrolled in the study (105 PWID and 104 MSM) during the data collection period, October-December 2019, (Figure 1).

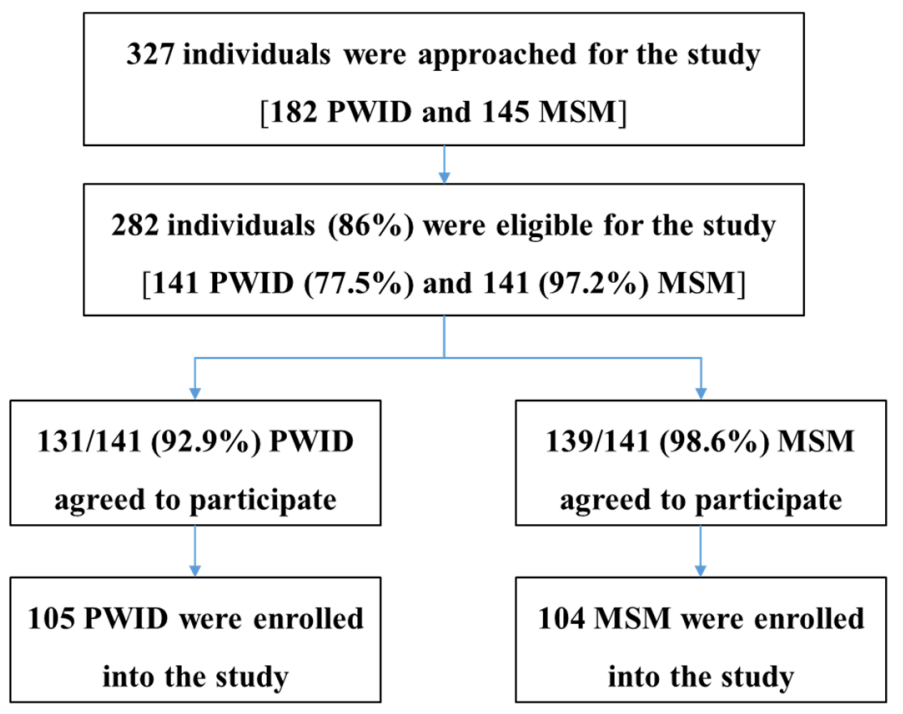

Figure 1. Flowchart of eligible individuals who were enrolled in the study (PWID: people who inject drugs and MSM: men who have sex with men).

The baseline characteristics of participants in the two groups are shown in Table 1. Compared to the MSM group, PWID were more likely to be older (median age: 45 vs. 22 years; $p<0.0001$ ), less educated (college, the highest level obtained in both groups: $1 \%$ in PWID vs. $87.5 \%$ in MSM, $p<0.0001$ ) and more unemployed (48.6\% in PWID vs. $2.9 \%$ in MSM; $p<0.0001)$. MSM were also more likely to be aware of self-testing than PWID ( $87.5 \%$ vs. $32.4 \% ; p<0.0001)$. Among two cohorts, only one participant in PWID had experience with self-testing, which was related to monitoring glucose as part of diabetes management. 
Table 1. Baseline demographic characteristics of study participants.

\begin{tabular}{|c|c|c|c|}
\hline Demographic Characteristics & PWID $(n=105)$ & $\operatorname{MSM}(n=104)$ & $p$ Value \\
\hline Age, years, median (range) & $45(31-62)$ & $22(18-26)$ & $<0.0001$ \\
\hline \multicolumn{4}{|l|}{ Sex, \% $(n)$} \\
\hline Male & $98.1(103)$ & $100.0(104)$ & 0.497 \\
\hline Female & $1.9(2)$ & 0 & \\
\hline Education, \% (n) & & & $<0.0001$ \\
\hline Primary school & $6.7(7)$ & 0 & \\
\hline Secondary school & $55.2(58)$ & 0 & \\
\hline High school & $37.1(39)$ & $12.5(13)$ & \\
\hline College & $1.0(1)$ & $87.5(91)$ & \\
\hline Occupation, \% (n) & & & $<0.0001$ \\
\hline Unemployed & $48.6(51)$ & $2.9(3)$ & \\
\hline Employed & $51.4(54)$ & $97.1(101)$ & \\
\hline Marital status, \% (n) & & & $<0.0001$ \\
\hline Married or living with a partner & $57.1(60)$ & $2.9(3)$ & \\
\hline Divorced, separated or widow & $17.1(18)$ & $1.0(1)$ & \\
\hline Unmarried & $25.7(27)$ & $96.2(100)$ & \\
\hline \multicolumn{4}{|l|}{$\begin{array}{l}\text { Self-reported exposures (ever) to any following risk factors } \\
\text { for HCV infection, } \%(n)\end{array}$} \\
\hline Injecting non-prescribed drugs & $100.0(105)$ & 0 & $<0.0001$ \\
\hline Sharing needles & $46.7(49)$ & 0 & $<0.0001$ \\
\hline Condomless anal intercourse & $6.7(7)$ & $100(104)$ & $<0.0001$ \\
\hline A surgical procedure & $11.4(12)$ & $15.4(16)$ & 0.424 \\
\hline A dental procedure & $27.6(29)$ & $45.2(47)$ & 0.009 \\
\hline Sharing shaving tools or toothbrushes & $25.7(27)$ & $28.8(30)$ & 0.643 \\
\hline Having a tattoo & $32.4(34)$ & $6.7(7)$ & $<0.0001$ \\
\hline Aware of the existence of self-tests performed at home, $\%(n)$ & $32.4(34)$ & $87.5(91)$ & $<0.0001$ \\
\hline Previous use of self-test, $\%(n)$ & $1.0(1)$ & 0 & 1.000 \\
\hline
\end{tabular}

\subsection{Usability of Self-Testing Performed by Participants}

HCVST procedures included completing 12 steps starting with opening the pouch and ending with interpreting the test result. Table 2 reports observer assessment of user mistakes completing steps overall and Table 3 reports observed difficulties and required assistance while self-testing.

Table 2. Observer assessment of mistakes (using product-specific checklist).

\begin{tabular}{|c|c|c|c|c|c|}
\hline \multirow{2}{*}{ Testing Steps } & \multicolumn{2}{|c|}{ PWID } & \multicolumn{2}{|c|}{ MSM } & \multirow{2}{*}{$p$ Value } \\
\hline & $n$ & $\%$ & $n$ & $\%$ & \\
\hline \multicolumn{6}{|l|}{ Pretesting } \\
\hline 1. Opening the pouch and taking all contents out & 0 & 0 & 0 & 0 & \\
\hline 2. Reading/using the instructions for use & 2 & 1.9 & 0 & 0 & 0.498 \\
\hline 3. Removing the test tube from the pack & 0 & 0 & 0 & 0 & \\
\hline 4. Removing the cap from the test tube & 2 & 1.9 & 0 & 0 & 0.498 \\
\hline 5. Sliding the test tube into the stand & 1 & 1 & 1 & 1 & 1.000 \\
\hline 6. Pouring the fluid from the test tube into the stand & 4 & 3.8 & 1 & 1 & 0.369 \\
\hline 7. Removing the test device from the pack & 0 & 0 & 0 & 0 & \\
\hline \multicolumn{6}{|l|}{ Conduct of Test } \\
\hline 8. Touching the test pad & 7 & 6.7 & 4 & 3.8 & 0.538 \\
\hline 9. Incorrectly collecting oral fluid sample * & 39 & 37.1 & 14 & 13.5 & 0.0001 \\
\hline 10. Wrong placing the test device in the test tube & 3 & 2.9 & 1 & 1 & 0.621 \\
\hline 11. Test device coming out of the tube while testing & 0 & 0 & 0 & 0 & \\
\hline 12. Not reading the result between 20 and $40 \mathrm{~min}$ & 4 & 3.8 & 2 & 1.9 & 0.683 \\
\hline \multicolumn{6}{|l|}{ Test Interpretation } \\
\hline 13. Wrong interpreting the test result ** & 21 & 20 & 1 & 1 & $<0.0001$ \\
\hline
\end{tabular}

* Correct manipulation is indicated in the instruction guide provided for participants: "Firmly put the flat pad to the gums and swab along the upper outer gum one time and along lower outer gum onetime"; ** Step considered correct if results read by the study participant agree with rereading by a trained staff. 
Table 3. Observer assessment of difficulties and steps requiring assistance.

\begin{tabular}{|c|c|c|c|c|c|c|}
\hline \multirow{3}{*}{ Testing Step, \% ( $n)$} & \multicolumn{3}{|c|}{ Difficulty Observed } & \multicolumn{3}{|c|}{ Assistance Provided } \\
\hline & PWID & MSM & \multirow{2}{*}{$p$} & PWID * & MSM & \multirow{2}{*}{$p$} \\
\hline & $n=105$ & $n=104$ & & $n=105$ & $n=104$ & \\
\hline Opening the pouch and taking all the contents out & $6.7(7)$ & $4.8(5)$ & 0.768 & $15.2(16)$ & $2.9(3)$ & 0.003 \\
\hline Reading/using the instructions for use & NA & NA & & $16.2(17)$ & $0(0)$ & $<0.0001$ \\
\hline Removing the cap from the test tube & $21.0(22)$ & $19.2(20)$ & 0.863 & $21.9(23)$ & $1(1)$ & $<0.0001$ \\
\hline Sliding the test tube into the stand & $11.4(12)$ & $1.9(2)$ & 0.01 & $17.1(18)$ & $0(0)$ & \\
\hline Collecting the oral fluid sample & NA & NA & & $21.9(23)$ & $0(0)$ & $<0.0001$ \\
\hline Placing the test device in the test tube & $1.0(1)$ & $1.9(2)$ & 0.621 & $15.2(16)$ & $1.9(2)$ & 0.0008 \\
\hline Interpreting the test result & $13.3(14)$ & $1.9(2)$ & 0.003 & $26.7(28)$ & $2.9(3)$ & $<0.0001$ \\
\hline
\end{tabular}

* Three participants had previous problems with their hand/s (injury and disability) and eight participants had poor eyesight that prompted request for assistance from study staff.

Both groups were generally able to self-test without errors. PWID were more likely to incorrectly interpret their HCVST result compared to MSM $(20 \%$ vs. $1 \% ; p<0.0001)$ (Table 2). The most common mistake observed was incorrect collection of the oral fluid specimen (37.1\% in PWID and $13.5 \%$ in MSM; $p=0.0001)$. The second most common mistake was touching the flat pad (used for collecting oral fluid) (6.7\% in PWID and 3.8\% in MSM; $p=0.538$.

For those needing assistance, the most difficult step was removing the cap from the developer solution (PWID: 21\% of PWID and MSM: 19.2\%) (Table 3). Sliding the developer solution into the stand was more challenging for PWID than MSM (11.4\% vs. $1.9 \% ; p=0.010)$. PWID needing assistance also had more difficulty interpreting their result correctly than did MSM (13.3\% vs. $1.9 \% ; p=0.003)$.

PWID also required more assistance, at all steps, than MSM (Tables 2 and 3). Among all participants with at least one mistake observed, PWID with and without study staff assistance made significantly more errors than MSM with or without study staff assistance. Any observed mistake, difficulty and provided assistance in the PWID group (62.9\% [66/105], 53.3\% [56/105] and 66.7\% [70/105], respectively) were significantly higher than in the MSM group (33.7\% [35/104], 28.8\% [30/104] and 17.3\% [18/104], respectively). The difference in proportions of any mistake, difficulty, and assistance between the two groups were statistically significant.

We also analyzed the association between demographic characteristics and levels of usability (mistakes, difficulties or provision of assistance). We found older age ( $>45$ years), lower educational level (primary or intermediate school), and marital status (married) were associated with higher proportion making either a mistake, having trouble or requiring assistance (Table 4). 
Table 4. Association between personal factors and usability of the hepatitis C virus (HCV) self-test.

\begin{tabular}{|c|c|c|c|c|c|c|c|c|c|}
\hline \multirow{2}{*}{ Demographic Characteristics } & \multicolumn{3}{|c|}{ Mistake } & \multicolumn{3}{|c|}{ Difficulty } & \multicolumn{3}{|c|}{ Assistance } \\
\hline & $n$ & $\%$ & $p$ & $n$ & $\%$ & $p$ & $n$ & $\%$ & $p$ \\
\hline \multicolumn{10}{|l|}{ Age, years, median (range) } \\
\hline$<22(\mathrm{~N}=51)$ & 21 & 41.2 & \multirow{3}{*}{0.0012} & 15 & 29.4 & \multirow{3}{*}{0.0030} & 9 & 17.6 & \multirow{3}{*}{$<0.0001$} \\
\hline $22-45(\mathrm{~N}=107)$ & 44 & 41.1 & & 40 & 37.4 & & 41 & 38.3 & \\
\hline$>45(\mathrm{~N}=51)$ & 36 & 70.6 & & 31 & 60.8 & & 38 & 74.5 & \\
\hline \multicolumn{10}{|l|}{ Sex, \% (n) } \\
\hline Male $(\mathrm{N}=207)$ & 48.8 & 101 & & 41.1 & 85 & & 42.0 & 87 & \\
\hline Female $(\mathrm{N}=2)$ & 0.0 & 0 & & 50.0 & 1 & & 50.0 & 1 & \\
\hline \multicolumn{10}{|l|}{ Educational level, \% ( $n$ ) } \\
\hline Primary school $(\mathrm{N}=7)$ & 4 & 57.1 & \multirow{4}{*}{0.0001} & 5 & 71.4 & \multirow{4}{*}{0.0010} & 6 & 85.7 & \multirow{4}{*}{$<0.0001$} \\
\hline Intermediate school $(\mathrm{N}=58)$ & 41 & 70.7 & & 33 & 56.9 & & 43 & 74.1 & \\
\hline Secondary school $(\mathrm{N}=62)$ & 27 & 43.5 & & 23 & 37.1 & & 22 & 35.5 & \\
\hline College $(\mathrm{N}=92)$ & 29 & 31.5 & & 25 & 27.2 & & 17 & 18.5 & \\
\hline \multicolumn{10}{|l|}{ Occupation, \% $(n)$} \\
\hline Unemployed $(\mathrm{N}=54)$ & 32 & 59.3 & \multirow{2}{*}{0.0875} & 25 & 46.3 & \multirow[b]{2}{*}{0.4641} & 29 & 53.7 & \multirow[b]{2}{*}{0.0651} \\
\hline Employed $(\mathrm{N}=155)$ & 69 & 44.5 & & 61 & 39.4 & & 59 & 38.1 & \\
\hline \multicolumn{10}{|l|}{ Marital status, \% $(n)$} \\
\hline Married or living with a partner $(\mathrm{N}=63)$ & 46 & 73.0 & \multirow{3}{*}{$<0.0001$} & 38 & 60.3 & \multirow{3}{*}{0.0011} & 39 & 61.9 & \multirow{3}{*}{$<0.0001$} \\
\hline Divorced, separated or widow $(\mathrm{N}=19)$ & 7 & 36.8 & & 6 & 31.6 & & 12 & 63.2 & \\
\hline Unmarried $(\mathrm{N}=127)$ & 48 & 37.8 & & 42 & 33.1 & & 37 & 29.1 & \\
\hline
\end{tabular}

\subsection{HCV Testing Results}

The HCV testing results performed by the participants (self-testing), re-interpreted by the study staff (rereading), and performed by study staff (retesting) in PWID and MSM are summarized in Figure 2. The self-testing results of the PWID group were $61.0 \%$ positive, $28.6 \%$ negative, $3.8 \%$ invalid, and $6.6 \%$ unable to interpret. Compared to the reference result determined with retesting performed by study staff, the positive rate with self-testing was lower in PWID group (61\% vs. $77.1 \% ; p=0.01$ ). In MSM group, $99 \%$ participants interpreted their results as negative and $1 \%$ as invalid while on rereading by study staff, one was read as a positive result. However, on retesting, the result was confirmed negative.

Table 5 shows the concordance of results between those interpreted by participants and study staff in both groups. In the PWID group, the Cohen's Kappa coefficient for inter-reader agreement was 0.77 . The concordance of inter-reader results in this group was $88.6 \%(93 / 105)$. There were five cases in which the participants interpreted the results as negative, but the results were all positive when reread by study staff. In another seven cases, where the participants were not able to interpret their results, the study staff reread and found four cases had positive results and three cases had invalid results. For interoperator agreement in PWID, the Cohen's Kappa coefficient was 0.61. The concordance of results between "self-testing" and "retesting" was $81.9 \%(86 / 105)$. There were eight cases in which the participants interpreted the results as negative, but they were all positive when the study staff retested. In the other four cases, which the participants interpreted as invalid results, two cases were positive and two were negative on retesting. There were seven cases in which the participants were not able to interpret their results, and all the results were positive when the seven participants were retested by staff. 


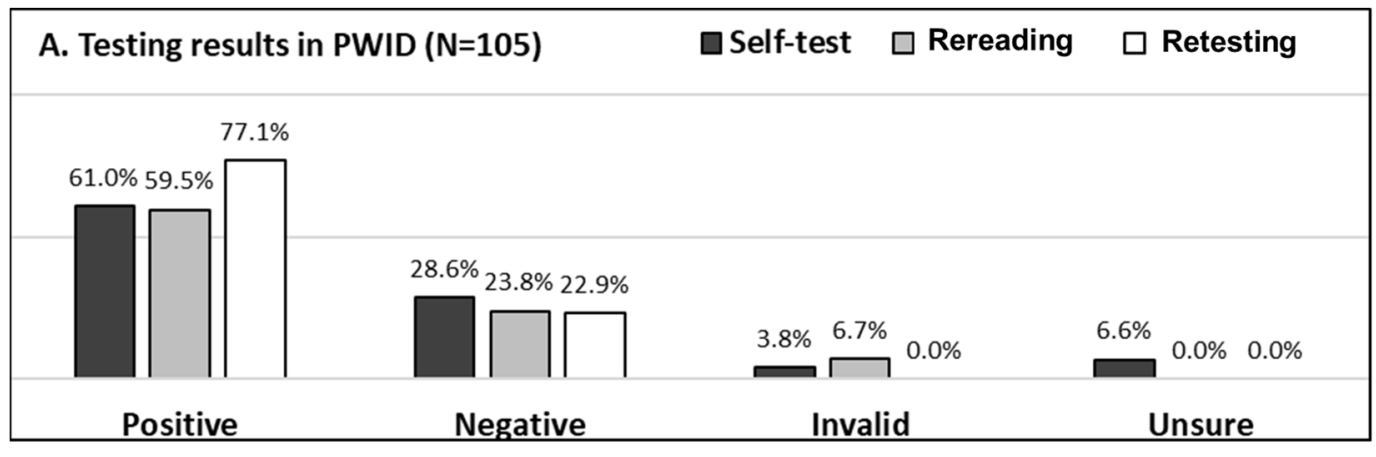

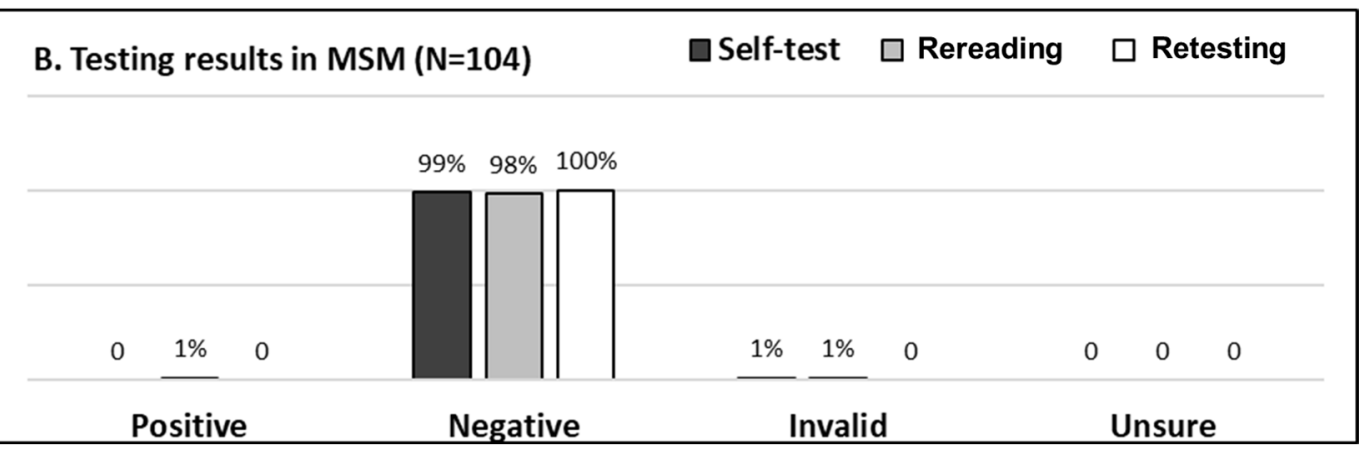

Figure 2. Summary of HCV testing results in PWID (A) and MSM (B) for participant self-testing, rereading by trained staff, and retesting performed by trained staff.

Table 5. The concordance of HCV testing results performed by the participants (self-testing), re-interpreted by the health care staff (rereading) and performed by the study staff (retesting) in PWID and MSM.

\begin{tabular}{|c|c|c|c|c|c|c|c|c|c|}
\hline \multirow{2}{*}{\multicolumn{2}{|c|}{ PWID (n) }} & \multicolumn{4}{|c|}{$\begin{array}{l}\text { Rereading by Trained Staff } \\
\text { (Inter-Reader) }\end{array}$} & \multicolumn{4}{|c|}{$\begin{array}{l}\text { Retesting by Trained Staff } \\
\text { (Inter-Operator) }\end{array}$} \\
\hline & & Positive & Negative & Invalid & Unsure & Positive & Negative & Invalid & Unsure \\
\hline \multirow{4}{*}{ 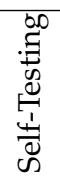 } & Positive & 64 & 0 & 0 & 0 & 64 & 0 & 0 & 0 \\
\hline & Negative & 5 & 25 & 0 & 0 & 8 & 22 & 0 & 0 \\
\hline & Invalid & 0 & 0 & 4 & 0 & 2 & 2 & 0 & 0 \\
\hline & Unsure & 4 & 0 & 3 & 0 & 7 & 0 & 0 & 0 \\
\hline \multicolumn{2}{|c|}{ Concordance (\%) } & \multicolumn{4}{|c|}{ Between self-testing and rereading: $88.6 \%$} & \multicolumn{4}{|c|}{ Between self-testing and retesting: $81.9 \%$} \\
\hline \multicolumn{2}{|c|}{ Cohen's Kappa } & \multicolumn{4}{|c|}{0.77} & \multicolumn{4}{|c|}{0.61} \\
\hline \multirow{2}{*}{\multicolumn{2}{|c|}{$\operatorname{MSM}(n)$}} & \multicolumn{4}{|c|}{$\begin{array}{l}\text { Rereading by Trained Staff } \\
\text { (Inter-Reader) }\end{array}$} & \multicolumn{4}{|c|}{$\begin{array}{l}\text { Retesting by Trained Staff } \\
\text { (Inter-Operator) }\end{array}$} \\
\hline & & Positive & Negative & Invalid & Unsure & Positive & Negative & Invalid & Unsure \\
\hline \multirow{4}{*}{ 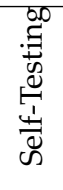 } & Positive & 0 & 0 & 0 & 0 & 0 & 0 & 0 & 0 \\
\hline & Negative & 1 & 102 & 0 & 0 & 0 & 103 & 0 & 0 \\
\hline & Invalid & 0 & 0 & 1 & 0 & 0 & 1 & 0 & 0 \\
\hline & Unsure & 0 & 0 & 0 & 0 & 0 & 0 & 0 & 0 \\
\hline \multicolumn{2}{|c|}{ Concordance $(\%)$} & \multicolumn{4}{|c|}{ Between self-testing and rereading: $99 \%$} & \multicolumn{4}{|c|}{ Between self-testing and retesting: 99\% } \\
\hline \multicolumn{2}{|c|}{ Cohen's Kappa } & \multicolumn{4}{|c|}{0.66} & \multicolumn{4}{|c|}{$0.99 *$} \\
\hline
\end{tabular}

In the MSM group, the Cohen's Kappa coefficient for inter-reader agreement was 0.66. The concordance of results between "self-testing" and "rereading" was 99\% (103/104). There was one case, in which the participant identified the result as negative, but it was determined as weak positive by a study staff member on rereading. For the inter-operator agreement, the Gwet's AC coefficient was 0.99. The concordance of results between "selftesting" and "retesting" was 99\% (103/104). There was one case in which the participant identified the result as invalid, but it was negative upon retesting by a study staff member. 
A total of 21 participants among 105 PWID (20\%) and one participant among 104 MSM $(1 \%)$ received discordant results between self-testing, rereading and retesting. All the discordant results from the 22 participants are listed in Table 6 and classified in four groups. The first group included six participants who incorrectly interpreted their results. All the results of these six participants were read by study staff as positive or weak positive, while after retesting the results of two participants changed from weak positive to negative. Among these six participants, one had poor eyesight, one did not understand the instruction, and two made mistakes during sample collection. The second group included four participants who correctly interpreted their result, but the self-test results were inconsistent with the retesting results performed by study staff. We observed that two of the four participants had incorrect sample collection. Five participants in the third group interpreted their results as invalid which were consistent with rereading by study staff; however, after retesting, three had negative results and all three of these had issues with sample collection. The other two had positive results after retesting but no mistake was observed. The fourth group listed seven participants who were unable to interpret their results even after assistance was provided to four out of seven participants. Four of the tests were read as positive by the study staff and all seven were positive after retesting. Among these seven participants, four had poor eyesight and one made mistakes while collecting the sample.

Table 6. Discordant results among self-testing, rereading (interpreted by study staff) and retesting (by the study staff) in PWID and MSM groups.

\begin{tabular}{|c|c|c|c|c|c|c|c|}
\hline Study ID & Self-Testing & Rereading & Retesting & $\begin{array}{l}\text { Touching } \\
\text { the Pad }\end{array}$ & $\begin{array}{l}\text { Collecting } \\
\text { Oral Fluid } \\
\text { Sample }\end{array}$ & $\begin{array}{l}\text { Assistance } \\
\text { to Interpret } \\
\text { Result }\end{array}$ & $\begin{array}{l}\text { Observations for } \\
\text { Discordant Results }\end{array}$ \\
\hline \multicolumn{8}{|c|}{ Wrong Result Interpretation by Participants $(n=6)$} \\
\hline PWID-011 & Negative & Positive & Positive & No & Correct & Yes & $\begin{array}{l}\text { Participant did not } \\
\text { understand the } \\
\text { instruction }\end{array}$ \\
\hline PWID-022 & Negative & Positive & Positive & No & Correct & No & \\
\hline PWID-048 & Negative & Weak Positive & Positive & No & Correct & Yes & $\begin{array}{c}\text { Participant had poor } \\
\text { eyesight }\end{array}$ \\
\hline PWID-082 & Negative & Positive & Positive & No & Incorrect & Yes & $\begin{array}{l}\text { Participant placed } \\
\text { device on the tongue } \\
\text { and swabbed the teeth }\end{array}$ \\
\hline PWID-097 & Negative & Weak positive & Negative & No & Incorrect & No & $\begin{array}{l}\text { Participant placed } \\
\text { device on the tongue }\end{array}$ \\
\hline MSM-062 & Negative & Weak Positive & Negative & No & Correct & Yes & \\
\hline \multicolumn{8}{|c|}{ Correct Result Interpretation by Participant but Retesting Result Differing from Self-Testing Result $(n=4)$} \\
\hline PWID-073 & Negative & Negative & Positive & No & Correct & No & \\
\hline PWID-075 & Negative & Negative & Positive & No & Incorrect & No & $\begin{array}{l}\text { Participant swabbed } \\
\text { the teeth }\end{array}$ \\
\hline PWID-105 & Negative & Negative & Weak positive & No & Correct & No & \\
\hline PWID-106 & Negative & Negative & Positive & No & Incorrect & No & $\begin{array}{l}\text { Participant swabbed } \\
\text { tongue and teeth }\end{array}$ \\
\hline \multicolumn{8}{|c|}{ Correct Interpretation of Invalid Results in Self-Testing $(n=5)$} \\
\hline PWID-010 & Invalid & Invalid & Positive & No & Correct & No & \\
\hline PWID-077 & Invalid & Invalid & Positive & No & Correct & No & \\
\hline PWID-017 & Invalid & Invalid & Negative & No & Incorrect & No & $\begin{array}{l}\text { Participant swabbed } \\
\text { on one gum only }\end{array}$ \\
\hline PWID-054 & Invalid & Invalid & Negative & No & Incorrect & No & $\begin{array}{l}\text { Participant did not use } \\
\text { the whole pad }\end{array}$ \\
\hline MSM-066 & Invalid & Invalid & Negative & Yes & Incorrect & No & $\begin{array}{l}\text { Participant did not } \\
\text { swab firmly enough }\end{array}$ \\
\hline
\end{tabular}


Table 6. Cont.

\begin{tabular}{|c|c|c|c|c|c|c|c|}
\hline Study ID & Self-Testing & Rereading & Retesting & $\begin{array}{l}\text { Touching } \\
\text { the Pad }\end{array}$ & $\begin{array}{c}\text { Collecting } \\
\text { Oral Fluid } \\
\text { Sample }\end{array}$ & $\begin{array}{l}\text { Assistance } \\
\text { to Interpret } \\
\text { Result }\end{array}$ & $\begin{array}{l}\text { Observations for } \\
\text { Discordant Results }\end{array}$ \\
\hline \multicolumn{8}{|c|}{ Unsure How to Interpret the Results in Self-Testing $(n=7)$} \\
\hline PWID-036 & Unsure & Positive & Positive & No & Correct & Yes & $\begin{array}{l}\text { Participant had poor } \\
\text { eyesight }\end{array}$ \\
\hline PWID-052 & Unsure & Positive & Positive & No & Correct & Yes & $\begin{array}{l}\text { Participant had poor } \\
\text { eyesight }\end{array}$ \\
\hline PWID-053 & Unsure & Positive & Positive & No & Correct & Yes & \\
\hline PWID-092 & Unsure & Positive & Positive & No & Incorrect & No & $\begin{array}{l}\text { Participant did not use } \\
\text { the whole pad }\end{array}$ \\
\hline PWID-043 & Unsure & Invalid & Positive & No & Correct & Yes & \\
\hline PWID-059 & Unsure & Invalid & Positive & No & Correct & No & $\begin{array}{l}\text { Participant had poor } \\
\text { eyesight }\end{array}$ \\
\hline PWID-064 & Unsure & Invalid & Positive & No & Correct & No & \\
\hline
\end{tabular}

\subsection{Acceptability and User Perspectives on HCVST}

After self-testing, participants provided their perspectives regarding perceptions of HCV self-test difficulty level at different steps (Figure 3).

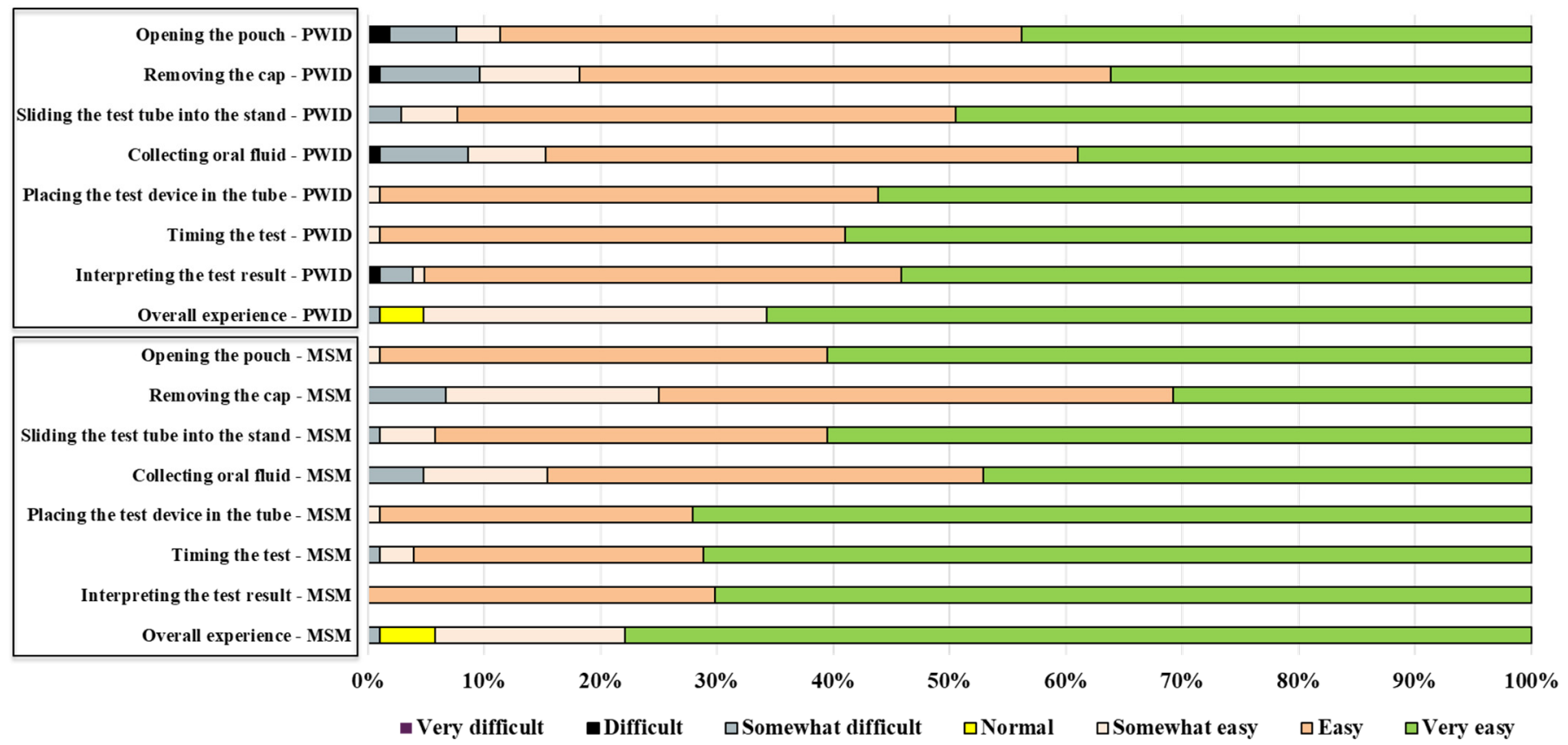

Figure 3. Participants' perceptions of HCV self-test usability at different steps.

Table 7 summarizes all the study outcomes. Acceptability was evaluated before and after self-testing. High acceptability in both groups was recorded $(>90 \%)$. The majority of the participants indicated a preference to test by themselves at home for $\mathrm{HCV}$ infection in the future $(69.5 \%$ in PWID and $76.9 \%$ in MSM, $p=0.275$ ) and their preferred sample type was oral fluid. Usability in MSM was significantly better than that in PWID, along with higher concordance of results in MSM compared to PWID. 
Table 7. Summary of acceptability, preference, usability and concordance results of HCV self-testing (HCVST).

\begin{tabular}{|c|c|c|c|}
\hline$\%(n)$ & PWID & MSM & $p$ Value \\
\hline Acceptability, \% (n) & $n=105$ & $n=104$ & \\
\hline \multicolumn{4}{|l|}{ Before self-testing } \\
\hline $\begin{array}{l}\text { The proportion of participants among eligible } \\
\text { subjects who agreed to participate and } \\
\text { perform HCV self-testing }\end{array}$ & $92.9(131 / 141)$ & $98.6(139 / 141)$ & 0.034 \\
\hline Ready to use HCV self-test if available & $98.1(103)$ & $100.0(104)$ & 0.498 \\
\hline \multicolumn{4}{|l|}{ After self-testing } \\
\hline Willing to use HCV test again & $91.4(96)$ & $98.1(102)$ & 0.058 \\
\hline $\begin{array}{l}\text { Willing to recommend the test to family and } \\
\text { friends }\end{array}$ & 99 (104) & $97.1(101)$ & 0.369 \\
\hline Taking the tests to family member/friend & $99.0(104)$ & $97.1(101)$ & 0.503 \\
\hline Preferences on HCVST, \% ( $n)$ & $n=105$ & $n=104$ & \\
\hline \multicolumn{4}{|l|}{ Preferred approach to test for HCV in the future } \\
\hline By myself at home & $69.5(73)$ & $76.9(80)$ & 0.275 \\
\hline By myself at a health center & $7.6(8)$ & $10.6(11)$ & 0.481 \\
\hline In a community center by a healthcare worker & $8.6(9)$ & $5.8(6)$ & 0.593 \\
\hline In a screening campaign & $0(0)$ & $2.9(3)$ & 0.121 \\
\hline \multicolumn{4}{|l|}{ Preferred sample type } \\
\hline Prefer oral fluid-based test & $79(83)$ & $67.3(70)$ & \\
\hline Prefer blood-based test & $10.5(11)$ & $26.9(28)$ & 0.416 \\
\hline No preference & $10.5(11)$ & $5.8(6)$ & \\
\hline \multicolumn{4}{|l|}{ Steps to take if results of self-test reactive } \\
\hline Contact healthcare facility & $95.2(100)$ & $83.7(87)$ & 0.006 \\
\hline Contact pharmacy & $1.9(2)$ & $1.9(2)$ & 1.000 \\
\hline Do a confirmatory test & $28.6(30)$ & $57.7(60)$ & $<0.0001$ \\
\hline $\begin{array}{l}\text { Seek advice from a family } \\
\text { member/community }\end{array}$ & $13.3(14)$ & $11.5(12)$ & 0.831 \\
\hline Do not know & $1.0(1)$ & $0(0)$ & 1.000 \\
\hline \multicolumn{4}{|l|}{ Knowledge about HCV treatment } \\
\hline Know that HCV can be cured & $64.8(68)$ & $55.8(58)$ & 0.205 \\
\hline $\begin{array}{l}\text { Know that there is a treatment but not sure } \\
\text { about the cure }\end{array}$ & $10.5(11)$ & $19.2(20)$ & 0.083 \\
\hline Not sure if there is treatment & $4.8(5)$ & $8.7(9)$ & 0.284 \\
\hline There is no treatment or cure & $0(0)$ & $1.9(2)$ & 0.246 \\
\hline \multicolumn{4}{|l|}{ Usability, \% $(n)$} \\
\hline $\begin{array}{l}\text { Correctly completing self-testing without any } \\
\text { mistake }\end{array}$ & $37.1(39)$ & $66.3(69)$ & $<0.0001$ \\
\hline Correctly collecting oral fluid & $62.9(66)$ & $86.5(90)$ & 0.0001 \\
\hline Correctly interpreting the self-test results & $80(84)$ & $99(103)$ & $<0.0001$ \\
\hline $\begin{array}{l}\text { Completing self-test procedure without } \\
\text { difficulty }\end{array}$ & $46.7(49)$ & $71.2(74)$ & 0.0004 \\
\hline $\begin{array}{l}\text { Completing self-test procedure without } \\
\text { assistance }\end{array}$ & $33.3(35)$ & $82.7(86)$ & $<0.0001$ \\
\hline \multicolumn{4}{|l|}{ Concordance of Results } \\
\hline \multicolumn{4}{|l|}{ Inter-reader agreement } \\
\hline Concordance, $\%(n)$ & $88.6 \%(93)$ & $99 \%(103)$ & 0.005 \\
\hline Kappa value & 0.77 & 0.66 & \\
\hline \multicolumn{4}{|l|}{ Inter-operator agreement } \\
\hline Concordance, $\%(n)$ & $81.9 \%(86)$ & $99 \%(103)$ & $<0.0001$ \\
\hline Kappa value & 0.61 & 0.99 & \\
\hline
\end{tabular}




\section{Discussion}

\subsection{Summary of Findings}

To our knowledge, this is one of the first studies to examine the acceptability and usability of HCVST in high-risk populations in a non-clinical setting. The study was conducted with PWID and MSM groups in a province of northern Vietnam. PWID were significantly older (median: 45 vs. 22 years) and had a lower educational level compared to MSM (Table 1).

\subsubsection{Usability}

The MSM group showed a significantly greater ability to correctly complete the selftesting procedure $(66.3 \%$ vs. $37.1 \%, p<0.0001)$, to complete HCVST without difficulty (71.2\% vs. $46.7 \%, p=0.0004)$ and without assistance $(82.7 \%$ vs. $33.3 \%, p<0.0001)$ compared to PWID (Table 7). In the PWID group, the collection of oral fluid samples and result interpretation were the two steps at which mistakes were most commonly observed ( $37.1 \%$ and 20\%, respectively) (Table 2). These two steps were also the ones most commonly requiring assistance $(21.9 \%$ and $26.7 \%)$. This was attributed to specific factors in these participants, including older age, lower educational background and health factors (poor eyesight, injuries to the hand, or mental condition). The difference in the proportion of participants observed to correctly complete steps, interpret results, and required assistance self-testing indicated that PWID may need more support self-testing, at least in the early days of implementation. Other HIVST studies have also reported that a common mistake in HIV self-testing was sample collection, and older individuals and those with lower levels of education were more prone to make errors. In a systematic review of reliability of the HIV rapid diagnostic test for self-testing [16], it was reported that the most common error that affected test performance was incorrect specimen collection. In another systematic review on acceptability of HIV self-testing, it was reported that accurate performance was associated with the level of education, and misinterpretation of photo results was associated with older age, although generally rare (4.9\%) [15].

\subsubsection{Concordance of Results}

Overall, there was good agreement between inter-reader and inter-operator in both groups. The concordance in the MSM group for both inter-reading and inter-operator (99\%) was higher than that in the PWID group ( $88.6 \%$ and $81.9 \%)$. With the inter-reading measure, the Cohen's Kappa coefficient in the PWID group (0.77) was relatively higher than that in MSM group (0.66). For inter-operator measure, the Cohen's Kappa coefficient in PWID (0.61) was lower than that in MSM group (0.99). The common mistakes we observed that affected the self-test results were incorrect sample collection, errors due to poor eyesight or difficulty in understanding the instruction. Assistance may be needed for those who have issues with reading or understanding the instructions. A demonstration video would be helpful in these cases. In 2019, Kimble et al. evaluated the performance of the OraQuick HCV Rapid Antibody Test in oral fluid when used by 95 patients (48 males and 47 female) for self-testing in the United States [21]. The authors reported that the test kit showed good performance when used by patients: sensitivity and specificity on self-collected oral fluid samples were $88.4 \% \%$ (95\% CI, 74.9-96.1) and 100\% (95\% CI, 93-100), respectively, when patients interpreted the test results. Sensitivity and specificity were $97.7 \%(95 \%$ CI, 88-99.9) and $98 \%$ (95\% CI, 89.6-100), respectively, when trained staff interpreted the result. The Cohen's Kappa coefficient was reported as 0.89 for inter-reading in this study.

\subsubsection{Acceptability}

There was high acceptability of HCV self-testing with more than $90 \%$ agreeing to participate in the study ( $92.9 \%$ of PWID and $98.6 \%$ of MSM). After the self-testing experience, the participants expressed a willingness to use an HCV self-test again or to introduce the self-test to family members and friends (Table 7). In 2018, in the first study to report on the acceptability of HCV self-testing performed by un-trained users [22], Guise et al. 
conducted qualitative rapid assessment to explore acceptability and key challenges of HCV self-testing among 22 study participants across three focus groups of PWID in the UK. The study showed potential acceptability but also revealed multiple concerns associated with self-testing such as poor access to confirmatory testing and care.

\subsection{The Performance and Applicability of OraQuick HCV Antibody Self-Test}

The clinical performance of the OraQuick HCV test has been evaluated and shown to be comparable to that of laboratory-based tests with both serum and oral fluid [23]. Overall, eight studies reported sensitivity and specificity of OraQuick (OraSure Technologies, PA, USA) with a total sample of 9024 . The sample size of these studies ranged from 172 to 2183 , sensitivities from $90 \%$ to $100 \%$, and specificities from $95 \%$ to $100 \%$ [23]. The pooled sensitivity and specificity were 98\% (95\% CI: 97\%-99\%) and 100\% (95\% CI: 90\%$100 \%$ ), respectively. Among studies that assessed individual oral RDTs, the eight studies showed that OraQuick ADVANCE ${ }^{\circledR}$ had a slightly higher sensitivity (98\%, 95\% CI: 9798\%) compared to the other oral brands (pooled sensitivity: 88\%, 95\% CI: 84-92\%). The performance characteristics of OraQuick had previously met the quality standards of RDT testing for oral specimen; therefore, in our study, we did not include testing using a reference assay to determine the sensitivity and specificity of the self-testing performed by the un-trained users. We focused mainly on the issues of acceptability and usability of HCV self-testing in two key populations, as these issues are critical in terms of feasibility and scale-up, and to improve the product to be more user-friendly.

Our findings have important implications for further work on self-testing. First, the higher proportion of PWID who experienced difficulties correctly performing the test, interpreting the results independently suggests that some individuals may require assistance, and face-to-face guidance or video demonstration would be useful. Second, we observed several steps where there were consistent difficulties, and we propose specific modifications to the product to improve the usability. The tube containing reagent was designed with a flip top cap, but participants often mistook them for screw cap tubes, which resulted in difficulty in opening the tube. The font size of the written instructions and the photo of result illustration were not large enough for those individuals with poor eyesight. The workflow of the testing procedure could be displayed in vertical order from the upper side down to the lower side in the instruction paper frame to prevent misunderstandings about the correct sequence of steps.

Other issues that need to be addressed are strategies to ensure prompt linkage and access to HCV viral load testing to confirm chronic HCV infection and need for treatment. At present, this represents a major barrier in access to care and treatment, especially among marginalized populations. There are also concerns that self-testing while promoting access to serological testing may result in sub-optimal linkage and uptake of treatment.

The implementation of HCV self-testing in Vietnam is important because key populations account for a significant proportion of the almost one million HCV-infected persons in the country. Based on 2013 estimates, there are around 271,506 PWID, 382,506 MSM and 71,936 sex workers in Vietnam, and all of them should be offered and have regular access to testing for HIV, hepatitis C and B, and sexually transmitted infections [24]. Self-testing represents a potential option to expand this access. Additionally, Vietnam has good experience in implementing HIV self-testing. The national guidelines for community-based HIV testing including HIV self-testing were approved by the Vietnam Ministry of Health in 2018 after one year of piloting in selected provinces [25]. HIV self-testing has now been scaled up country wide. The feasibility and effectiveness of HIV self-testing in case finding and linkages to care has been reported [20]. This represents a strong foundation for the implementation of HCV self-testing once test kits are made available for communities.

This study presented some limitations. The convenience sampling may present a selection bias in PWID and MSM at the selected local sites. Given the low positive rate of anti-HCV in the MSM group, a larger sample size for MSM would be better to assess the concordance of the test results. Also, female sex workers as one of the key populations was 
not included in this study. Finally, assessment for dual risk and screening for high risk for $\mathrm{HCV}$ acute infection were not conducted in this study.

\section{Conclusions}

This study shows a favorable experience with an oral fluid HCV self-test in two key populations, PWID and MSM. Self-testing for HCV may also be a good option for other populations to learn their HCV status. Additional assistance such as demonstration videos or support from health staff or peer educators during the self-testing process may be needed for individuals who conduct self-testing for the first time or who have difficulty in following instructions for users. The study findings provide important information for development of HCVST that may facilitate scale-up of HCV screening and improve coverage of $\mathrm{HCV}$ treatment. This emerging evidence provides a strong foundation to support further studies to inform global, regional, and national HCV testing policy and expanded access to HCV testing.

Author Contributions: Formal analysis, L.T.N. and V.T.T.N.; investigation, L.T.N., V.T.T.N., K.A.L.A., M.B.T. and T.T.M.T.; writing—original draft, L.T.N. and V.T.T.N.; writing—review and editing, V.T.T.N., L.T.N., C.J., M.S.J., E.I.R., P.E. and K.P. All authors have read and agreed to the published version of the manuscript.

Funding: This research was funded by Unitaid through WHO, grant number 2016-10-WHO.

Institutional Review Board Statement: The study was conducted according to the guidelines of the Declaration of Helsinki, and approved by Review Board in Bio-medical Research at National Institute of Hygiene and Epidemiology, Hanoi, Vietnam on 30 July 2019 (IRB-VN01057/IORG 0008555, number NIHE IRB-20/2019).

Informed Consent Statement: Informed consent was obtained from all subjects involved in the study.

Data Availability Statement: The data presented in this study are available on request from the corresponding author. The data are not publicly available due to confidentiality.

Acknowledgments: The authors would like to thank the leaders and the health staff in the Center for Disease Control and the PWID and MSM peer educators in Thai Nguyen province for their support of the study. We also would like to thank Emmanuel Fajardo and Elkin Bermudez Aza for their valuable advice on data analysis. The study was funded by Unitaid through WHO.

Conflicts of Interest: The authors declare no conflict of interest. The sponsors had no role in the design, execution, interpretation, or writing of the study. The contents in this article are those of the authors and do not necessarily reflect the view of the World Health Organization.

\section{References}

1. World Health Organization. Global Hepatitis Report 2017; World Health Organization: Geneva, Switzerland, 2017.

2. World Health Organization. Global Health Sector Strategy on Viral Hepatitis 2016-2021; World Health Organization: Geneva, Switzerland, 2016.

3. Ho, S.Y.; Su, L.H.; Sun, H.Y.; Huang, Y.S.; Chuang, Y.C.; Huang, M.H.; Liu, W.C.; Su, Y.C.; Lin, P.H.; Chang, S.Y.; et al. Trends of recent hepatitis $C$ virus infection among HIV-positive men who have sex with men in Taiwan, 2011-2018. EClinicalMedicine 2020, 24, 100441. [CrossRef]

4. World Health Organization Regional Office for the Western Pacific. Viral Hepatitis Situation and Response in Viet Nam; World Health Organization: Geneva, Switzerland, 2019.

5. Zhang, L.; Celentano, D.D.; Le Minh, N.; Latkin, C.A.; Mehta, S.H.; Frangakis, C.; Ha, T.V.; Mo, T.T.; Sripaipan, T.; Davis, W.W.; et al. Prevalence and correlates of HCV monoinfection and HIV and HCV coinfection among persons who inject drugs in Vietnam. Eur. J. Gastroenterol. Hepatol. 2015, 27, 550-556. [CrossRef]

6. Nguyen Truong, T.; Laureillard, D.; Lacombe, K.; Duong Thi, H.; Pham Thi Hanh, P.; Truong Thi Xuan, L.; Chu Thi, N.; Luong Que, A.; Vu Hai, V.; Nagot, N.; et al. High Proportion of HIV-HCV Coinfected Patients with Advanced Liver Fibrosis Requiring Hepatitis C Treatment in Haiphong, Northern Vietnam (ANRS 12262). PLoS ONE 2016, 11, e0153744. [CrossRef] [PubMed]

7. Nadol, P.; O'Connor, S.; Duong, H.; Mixson-Hayden, T.; Tram, T.H.; Xia, G.L.; Kaldor, J.; Law, M.; Nguyen, T. High hepatitis $C$ virus (HCV) prevalence among men who have sex with men (MSM) in Vietnam and associated risk factors: 2010 Vietnam Integrated Behavioural and Biologic Cross-Sectional Survey. Sex. Transm. Infect. 2016, 92, 542-549. [CrossRef] [PubMed] 
8. Berto, A.; Day, J.; Van Vinh Chau, N.; Thwaites, G.E.; My, N.N.; Baker, S.; Darton, T.C. Current challenges and possible solutions to improve access to care and treatment for hepatitis C infection in Vietnam: A systematic review. BMC Infect. Dis. 2017, 17, 260. [CrossRef] [PubMed]

9. Dunford, L.; Carr, M.J.; Dean, J.; Waters, A.; Nguyen, L.T.; Ta Thi, T.H.; Thi, L.A.; Do, H.D.; Thi, T.T.; Nguyen, H.T.; et al. Hepatitis $C$ virus in Vietnam: High prevalence of infection in dialysis and multi-transfused patients involving diverse and novel virus variants. PLoS ONE 2012, 7, e41266. [CrossRef] [PubMed]

10. Pawlotsky, J.M. New hepatitis C therapies: The toolbox, strategies, and challenges. Gastroenterology 2014, 146, 1176-1192. [CrossRef] [PubMed]

11. Feeney, E.R.; Chung, R.T. Antiviral treatment of hepatitis C. BMJ 2014, 348, g3308. [CrossRef] [PubMed]

12. World Health Organization. Guidelines on Hepatitis B and C Testing; World Health Organization: Geneva, Switzerland, 2017.

13. Johnson, C.C.; Kennedy, C.; Fonner, V.; Siegfried, N.; Figueroa, C.; Dalal, S.; Sands, A.; Baggaley, R. Examining the effects of HIV self-testing compared to standard HIV testing services: A systematic review and meta-analysis. J. Int. AIDS Soc. 2017, $20,21594$. [CrossRef] [PubMed]

14. Zhang, C.; Li, X.; Brecht, M.L.; Koniak-Griffin, D. Can self-testing increase HIV testing among men who have sex with men: A systematic review and meta-analysis. PLoS ONE 2017, 12, e0188890. [CrossRef] [PubMed]

15. Krause, J.; Subklew-Sehume, F.; Kenyon, C.; Colebunders, R. Acceptability of HIV self-testing: A systematic literature review. BMC Public Health 2013, 13, 735. [CrossRef] [PubMed]

16. Figueroa, C.; Johnson, C.; Ford, N.; Sands, A.; Dalal, S.; Meurant, R.; Prat, I.; Hatzold, K.; Urassa, W.; Baggaley, R. Reliability of HIV rapid diagnostic tests for self-testing compared with testing by health-care workers: A systematic review and meta-analysis. Lancet HIV 2018, 5, e277-e290. [CrossRef]

17. Pant Pai, N.; Sharma, J.; Shivkumar, S.; Pillay, S.; Vadnais, C.; Joseph, L.; Dheda, K.; Peeling, R.W. Supervised and unsupervised self-testing for HIV in high- and low-risk populations: A systematic review. PLoS Med. 2013, 10, e1001414. [CrossRef] [PubMed]

18. World Health Organization. Guidelines on HIV Self-testing and Partner Notification: Supplement to Consolidated Guidelines on HIV Testing Services; World Health Organization: Geneva, Switzerland, 2016.

19. World Health Organization. WHO List of Prequalified In Vitro Diagnostic Products. Available online: https://www.who.int/ diagnostics_laboratory / evaluations/190121_prequalified_product_list.pdf?ua=1 (accessed on 6 November 2020).

20. Nguyen, V.T.T.; Phan, H.T.T.; Kato, M.; Nguyen, Q.; Le Ai, K.A.; Vo, S.H.; Thanh, D.C.; Baggaley, R.C.; Johnson, C.C. Communityled HIV testing services including HIV self-testing and assisted partner notification services in Vietnam: Lessons from a pilot study in a concentrated epidemic setting. J. Int. AIDS Soc. 2019, S3, e25301. [CrossRef]

21. Kimble, M.M.; Stafylis, C.; Treut, P.; Saab, S.; Klausner, J.D. Clinical evaluation of a hepatitis C antibody rapid immunoassay on self-collected oral fluid specimens. Diagn. Microbiol. Infect. Dis. 2019, 95, 149-151. [CrossRef] [PubMed]

22. Guise, A.; Witzel, T.C.; Mandal, S.; Sabin, C.; Rhodes, T.; Nardone, A.; Harris, M. A qualitative assessment of the acceptability of hepatitis $C$ remote self-testing and self-sampling amongst people who use drugs in London, UK. BMC Infect. Dis. 2018, 18, 281. [CrossRef]

23. Tang, W.; Chen, W.; Amini, A.; Boeras, D.; Falconer, J.; Kelly, H.; Peeling, R.; Varsaneux, O.; Tucker, J.D.; Easterbrook, P. Diagnostic accuracy of tests to detect Hepatitis C antibody: A meta-analysis and review of the literature. BMC Infect. Dis. 2017, 17 (Suppl. 1), 695. [CrossRef]

24. PEPFAR. VIETNAM Country/Regional Operational Plan (COP/ROP) 2016: Strategic Direction Summary. 2016. Available online: https:/ / mz.usembassy.gov/wp-content/uploads/sites/182/2017/04/PEPFAR-cop16-Guidance.pdf (accessed on 20 May 2020).

25. Vietnam Ministry of Health. Community-Based Counselling and Testing Guidelines; Ministry of Health: Hanoi, Vietnam, 2018. 